Draft version June 13, 2018

Preprint typeset using LATEX style emulateapj v. 08/22/09

\title{
THE DIFFUSE SOFT EXCESS EMISSION IN THE COMA CLUSTER FROM THE ROSAT ALL-SKY SURVEY
}

\author{
M. Bonamente ${ }^{1,2}$, R. Lieu ${ }^{1}$, And E. Bulbul ${ }^{1}$ \\ Draft version June 13, 2018
}

\begin{abstract}
ROSAT All-Sky Survey data near the North Galactic Pole was analyzed in order to study the largescale distribution of soft X-ray emission from the Coma cluster. These ROSAT data constitute the only available X-ray observations of Coma that feature an in situ - temporally and spatially contiguous - background, with unlimited and continuous radial coverage. These unique characteristics of the ROSAT All-Sky Survey data are used to deliver a final assessment on whether the soft excess previously detected in the Coma cluster is due to background subtraction errors, or not. This paper confirms the presence of soft X-ray excess associated with Coma, and reports the detection of $1 / 4 \mathrm{keV}$ band excess out to $5 \mathrm{Mpc}$ from the cluster center, the largest soft excess halo discovered to date. We propose that the emission is related to filaments that converge towards Coma, and generated either by non-thermal radiation caused by accretion shocks, or by thermal emission from the filaments themselves.

Subject headings: galaxies: clusters: individual (Coma); cosmology: large-scale structure of universe
\end{abstract}

\section{INTRODUCTION}

X-ray emission from galaxy clusters is due primarily to a hot virialized plasma at a temperature $k T \sim$ $10^{7}-10^{8} \mathrm{keV}$ that fills the intra-cluster medium (ICM). Evidence for an additional emission component was initially discovered by Lieu et al. (1996a,b) in the extreme ultra-violet $(h \nu \sim 0.1 \mathrm{keV})$ with EUVE observations of the Virgo and Coma clusters, and then confirmed with several other instruments, notably in the ROSAT $1 / 4$ $\mathrm{keV}$ band (see Durret et al. 2008 for a recent review of the literature; detections in Coma include Bowver et al. 2004, Finoguenov et al. 2003, Bonamente et al. 2003, Nevalainen et al. 2003, Bowyer et al. 1999). The excess emission is usually modeled as an additional thermal component of lower temperature $\left(k T \sim 10^{6}-10^{7}\right.$ $\mathrm{keV}$ ) or as a non-thermal power law. An additional thermal model may arise from cooler gas inside the cluster (Lieu et al. 1996b), possibly in pressure equilibrium as in the Cheng et al. (2005) model, or from warm filaments seen in projection towards clusters (e.g., Mittaz et al. 2004; Bonamente et al. 2005). A non-thermal power law is indicative of relativistic electrons that scatter the CMB photons and emit by Compton scattering (e.g., Sarazin \& Lieu 1998; Lieu et al. 1999). Given the limited spectral resolution of the current CCD detector technology, it has not been possible to prove conclusively which additional model is a better fit to the data (see, e.g., Nevalainen et al. 2003; Bonamente et al. 2005; Werner et al. 2007).

In the main X-ray band $(\sim 0.5-10 \mathrm{keV})$, cluster spectra in annular regions are typically well fit by singletemperature models. Recently, it has become evident that the best-fit temperature of the hot plasma is banddependent, with a trend of lower temperatures when lower-energy bands are used in the fit (Nevalainen et al. 2003; Cavagnolo et al. 2008; Bonamente et al. 2007); this behavior is naturally explained by the presence of an ad-

\footnotetext{
${ }^{1}$ Physics Department, University of Alabama in Huntsville, Huntsville, Al 35899

2 NASA National Space and Technology Center, Huntsville, Al 35899
}

ditional soft component.

In the absence of conclusive spectral evidence on the origin of the excess emission, clues can be found from the spatial distribution of the excess. The excess emission typically increases with radius, with respect to the hot ICM (e.g., Lieu et al. 1999; Bonamente et al. 2001a), indicating that the excess component is likely unrelated to the hot ICM. Attempts at resolving the emission have so far been unsuccessful (e.g. Bonamente et al. 2003), and thus a truly diffuse origin for the excess emission is favored. To date, the excess emission was detected out to a maximum radius of $1.7 \mathrm{Mpc}$ (Bonamente et al. 2003), and typically at radii $\leq 0.5-1 \mathrm{Mpc}$; at these radii, the hot plasma has a density of $\simeq 10^{-4}-10^{-5} \mathrm{~cm}^{-3}$, and can support $\sim \mu \mathrm{G}$ magnetic fields that give rise to radio halos (e.g. Feretti et al. 2001; Brunetti et al. 2007; Clarke \& Ensslin 2006). Therefore, a non-thermal origin of the excess can be justified.

Detection of the excess emission is particularly sensitive to the background subtraction process. The soft $\mathrm{X}$-ray sky is known to have gradients on the scales of degrees (e.g., Snowden et al. 1997), requiring that the background is estimated from a region that is spatially contiguous to the cluster. Moreover, temporal variability can be induced by Solar flares, which cause chargeexchange radiation that varies on time scales of hours; the most accurate background subtraction is therefore performed when a simultaneous background measurement is available, e.g., from peripheral regions of the detector. Such time- and space-contiguous background was used in the early EUVE and ROSAT works, thanks to the large field of view of those instruments. In the case of XMM-Newton and Chandra, the local background is only available for high-redshift clusters of smaller angular size. An incorrect background subtraction caused by time variability was shown by Takei et al. (2008) to be the reason for an earlier claim of detection of O VII emission lies associated with the soft excess emission. In the case of the Coma cluster, even the 1 degree radius of the $P S P C$ emission is entirely filled by cluster emission, and therefore a time-contiguous background was not avail- 
able in our earlier analysis based on pointed observation (Bonamente et al. 2003).

In this paper we analyze ROSAT All-Sky Survey data in the direction of the Coma cluster. Thanks to the allsky nature of the observations, these data are the only observations of Coma to feature a background that is spatially and temporally contiguous, and with unlimited radial coverage. We therefore aim to show that excess emission is present when this local background is used, and determine the maximum radius of detection of the excess emission. This paper is structured in the following way: in Section 2 we describe the ROSAT All-Sky Survey data, the data analysis and the detection of the soft excess emission, in Section 3 we interpret the emission using thermal and non-thermal models, and in Section 4 we discuss our results and present our conclusions.

\section{DATA REDUCTION AND ANALYSIS}

\subsection{The ROSAT All-Sky Survey data}

The angular resolution of the ROSAT X-ray telescope in combination with the $P S P C$ camera is approximately $1.8^{\prime}(50 \%$ encircled energy radius averaged over the field of view), and the energy resolution is approximately $\Delta E / E=0.43(E / 0.93 \mathrm{keV})^{-0.5} \mathrm{FWHM}$, corresponding to a $\Delta E \sim 0.2 \mathrm{keV}$ in the $1 / 4 \mathrm{keV}$ band, and $\Delta E \sim 0.5$ $\mathrm{keV}$ at $1.5 \mathrm{keV}$. The field of view of $P S P C$ is circular with approximately 1 degree radius. During the first year of operation, ROSAT performed an all-sky X-ray survey, which included an exposure of $\sim 850$ seconds in the direction of the Coma cluster (Briel et al. 1992). Snowden et al. (1997) analyzed the ROSAT All-Sky Survey (RASS) and provided maps on the diffuse soft X-ray emission in seven bands (R1 through R7), with linear pixel of size $12^{\prime}$. The $\mathrm{R} 2$ band is the softest band with reliable calibration ( $90 \%$ of peak response $0.14-0.284 \mathrm{keV}$ ), and band R7 (1.05-2.04 keV) is the highest energy band available with the $P S P C$ detector.

The maps exclude bright point-like sources identified from the RASS data themselves, using a flux threshold for which the RASS source catalog is complete over $90 \%$ of the sky. In the R1+R2 band, the flux threshold is 0.025 counts $\mathrm{s}^{-1}$, and in the $\mathrm{R} 6+\mathrm{R} 7$ band it is 0.020 counts s $^{-1}$ (Snowden et al. 1997). This choice resulted in the exclusion of the central pixel of the Coma cluster in the R2 band, which appears point-like at the resolution of these data. The units of the emission maps are $10^{-6}$ PSPC counts $\mathrm{s}^{-1} \operatorname{arcmin}^{-2}$ (a detector-specific measure of the intensity of radiation, proportional to the surface brightness), and take into account the effects of vignetting, different exposure times of a given sky region, detector artifacts, obscuration by the window support structure, and other efficiency variations. The maps of the diffuse emission are complemented by matching maps of the estimated uncertainty in the intensity, needed in order to determine the statistical significance of the emission above the local background (Snowden et al. 1997). ${ }^{3}$

These X-ray data represent a unique opportunity for the detection of diffuse emission associated with the

3 The maps of the diffuse X-ray emission can be found at http://www.xray.mpe.mpg.de/rosat/survey/sxrb/12/ass.html The $\mathrm{R} 2$ and $\mathrm{R} 7$ bands used in this work are g000p90r2b120pm.fits and g000p90s2b120pm.fits for R2 band, and g000p90r7b120pm.fits and g000p90s7b120pm.fits for R7 band.
Coma cluster. These are in fact the only observations that feature both a large radial coverage and a simultaneous background measurement. The latter feature is especially significant, in an effort to avoid background subtraction problems caused by the charge-exchange radiation induced by solar activity (Takei et al. 2008). Owing to the scanning mode in which ROSAT was operated during the survey, any enhancement caused by solar windinduced emission will be present in both cluster and background regions. No other X-ray mission has performed a survey of the X-ray sky, or has a field of view which covers such a large area of the sky. The pointed ROSAT AllSky Survey observations of Coma, which we analyzed in a previous paper (Bonamente et al. 2003), covered only a region of 1 degree radius, and thus could not investigate the emission beyond that distance, or with a local background.

\subsection{Calibration accuracy of the ROSAT PSPC}

Initial calibration of the ROSAT PSPC was performed on the ground (Pfeffermann et al. 1987). Of particular interest to the results of this paper is the calibration of the effective area in the R2 band. Snowden et al. (1995) compared $P S P C$ observations of the diffuse soft X-ray background with observations from the Wisconsin survey (e.g. McCammon et al. 1983; Garmire et al. 1992); the comparison revealed that the $P S P C$ effective area was well calibrated within a $10 \%$ uncertainty. More recently, Beuermann et al. (2006) compared the extremeultraviolet and soft X-ray fluxes of three standard candles observed by the Chandra LETG, EUVE and ROSAT $P S P C$, and found that the agreement between the various instruments indicate that the $P S P C$ effective area is calibrated within a few $\%$ error. These results indicate that the uncertainties in the $\mathrm{R} 2$ band effective area are of the order of few percent; as it will be shown in the following, this level of uncertainty will not affect any of the results presented in this paper.

\subsection{Images and radial profiles of the X-ray surface brightness}

In Figure 1 we show the diffuse surface brightness in a region of 400 square degrees around the Coma cluster. The R2 band map features the northern edge of the North Polar Spur in the bottom right quadrant of panel (a); the two black pixels indicate the location of two point-like features removed by the Snowden et al. (1997) data reduction. The R7 band image indicates that the 1-2 keV local background in the neighborhood of Coma does not feature any large-scale gradients.

We obtained azimutally averaged radial profiles of the surface brightness in the two bands (Figure 2), centered at R.A $=12^{h} 59^{\prime} 50^{\prime \prime}$, Dec. $=+27^{\circ} 58^{\prime} 59^{\prime \prime}$, located at the center of Figure 1. 4 Analysis of the R2 profile in Figure 2(a) shows that the North Polar Spur region is likely to affect the level of emission at distances $\geq 6^{\circ}$ from Coma. We therefore estimate the local background in two ways: as the average of the $4-6^{\circ}$ region, and as the average of the $4-10^{\circ}$ region (Table 1). Given that the two estimates of the background are statistically consistent with one another in each band, we use the $4-6^{\circ}$

4 This position corresponding to pixel $(247.80,251.08)$ in the Snowden maps. 

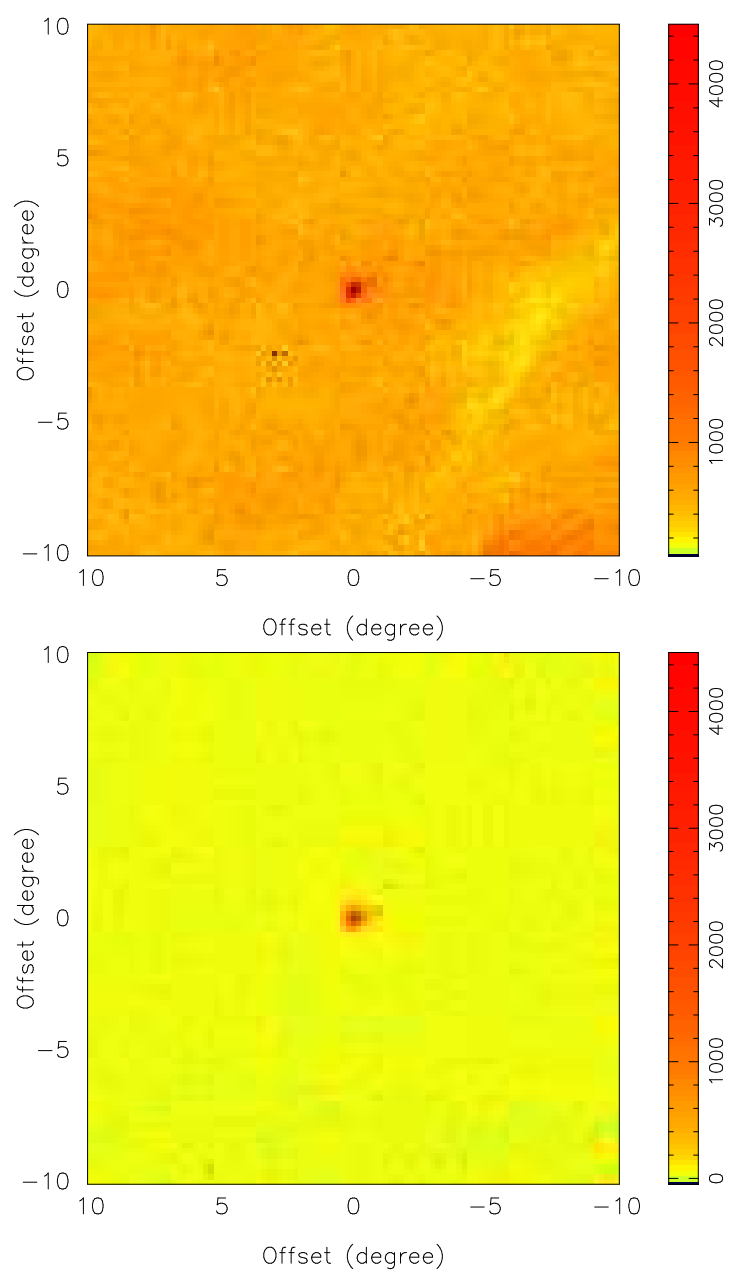

FIG. 1.- Images of the diffuse emission of Coma in bands R2 (a) and R7 (b) . Units of the emissions are $10^{-6}$ PSPC counts s ${ }^{-1}$ $\operatorname{arcmin}^{-2}$.

background in the following analysis; all results presented in this paper are unchanged if the $4-10^{\circ}$ background is used instead. The choice of $4^{\circ}$ as the minimum distance is motivated by the fact that the emission appears to fade into the background between 3 and 4 degrees, consistent with the fact that Coma is not known to emit $\mathrm{X}$-rays at such large distances. For a Hubble constant of $H_{0}=73 \mathrm{~km} \mathrm{~s}^{-1} \mathrm{Mpc}^{-1}, 1$ degree corresponds to 1.67 $\mathrm{Mpc}$ at the redshift of the Coma cluster $(z=0.024)$.

The radial profiles indicate the $1-2 \mathrm{keV}$ emission reaches the local background at a radius of $\sim 2^{\circ}$, while the softer R2 band has emission above the local background out to (at least) $3^{\circ}$. The radial profiles therefore indicate that the Coma cluster has an additional soft emission component at large radii. Measurements of the virial radius of Coma were recently obtained by weak lensing measurements (Kubo et al. 2007, $r_{200}=2.7 \pm 0.3$ Mpc for $\left.H_{0}=73 \mathrm{~km} \mathrm{~s}^{-1} \mathrm{Mpc}^{-1}\right)$, galaxy kinematics (Eokas \& Mamon 2003, $r_{v}=2.8 \pm 0.8 \mathrm{Mpc}$ for $H_{0}=73$ $\mathrm{km} \mathrm{s}^{-1} \mathrm{Mpc}^{-1}$ ) and redshift survey of cluster galaxies (Geller et al. 1999, $r_{200} \simeq 2.1 \mathrm{Mpc}$ for $H_{0}=73$ $\left.\mathrm{km} \mathrm{s}^{-1} \mathrm{Mpc}^{-1}\right)$. These measurements are in agreement with earlier X-ray measurements by Briel et al. (1992, based on these ROSAT data) and Hughes (1989, based on Einstein data), which detected X-ray emission at ra-
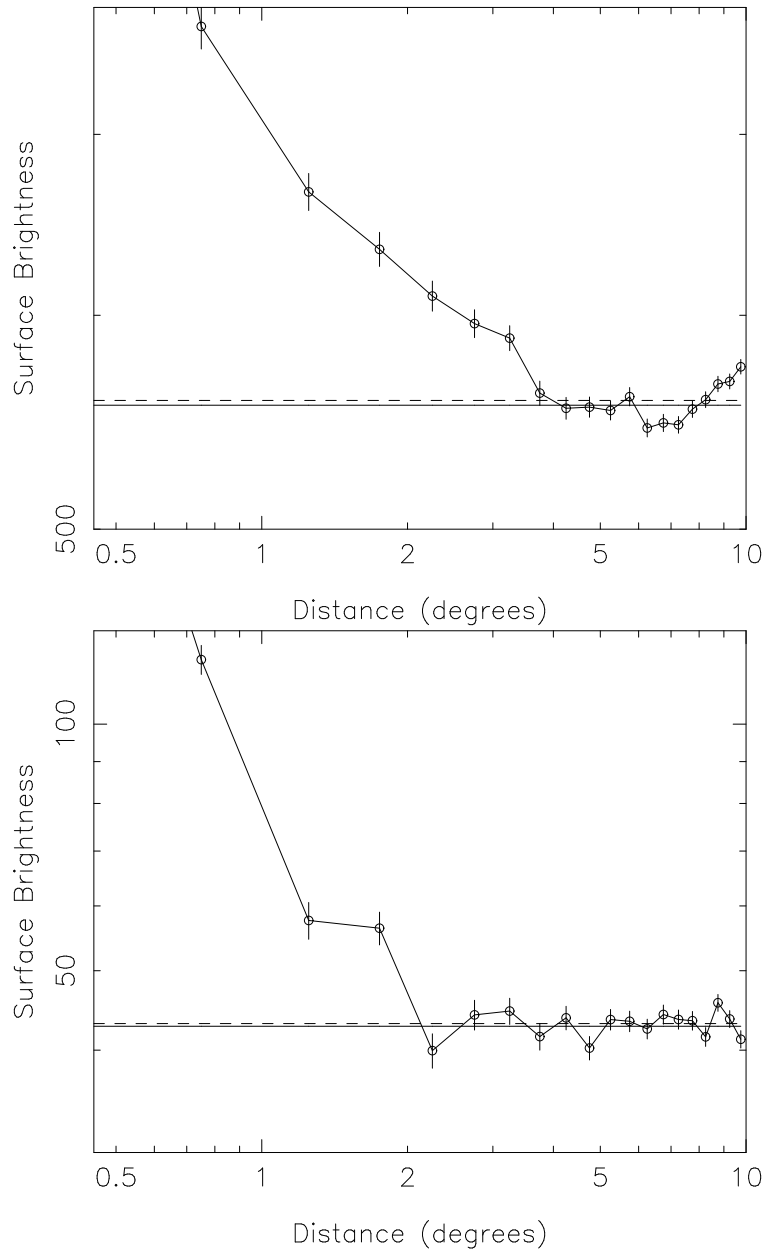

FIG. 2.- Radial profiles of the diffuse emission near Coma in R2 (a) and R7 band (b). Units of the surface brightness are $10^{-6}$ PSPC counts $\mathrm{s}^{-1}$ arcmin $^{-2}$.

dial distance of $\leq 100$ arcmin. The present detection of soft X-ray emission out to (at least) $3^{\circ}$ or $5 \mathrm{Mpc}$, therefore tracks the soft X-ray emission out to the virial radius, and also constitutes the largest continuous halo of $\mathrm{X}$-ray emission detected to date in any galaxy cluster.

\subsection{The soft $X$-ray excess emission}

Futher analysis of the soft emission must take into account the contribution of the hot intra-cluster medium to the R2 band emission. Given the limited spectral resolution and narrow bandpass $(\sim 0.2-2 \mathrm{keV})$, the ROSAT All-Sky Survey PSPC data are not ideal for the determination of hot plasma temperatures. We therefore rely on other X-ray studies of Coma, which consistently measure a plasma temperature of $k T \simeq 8 \mathrm{keV}$ in the central $20^{\prime}$ (e.g., Arnaud et al. 2001; Lutovinov et al. 2008). At larger radii, Finoguenov et al. (2001) measure temperatures in the range of $15-3 \mathrm{keV}$ for regions between $0.5-$ $1.5^{\circ}$ from the cluster center, consistent with the decrease of temperature at large radii observed in other clusters (Vikhlinin et al. 2006).

In order to estimate the hot ICM contribution to the R2 band, we assume that the average plasma temperature has the distribution shown in Table 2, decreasing from $8 \mathrm{keV}$ to $2 \mathrm{keV}$ between the center and the outskirts. 


\begin{tabular}{l|cc}
\hline & $\mathrm{R} 2$ & $\mathrm{R} 7$ \\
\hline $4-6^{\circ}$ & $555.7 \pm 2.3$ & $42.8 \pm 0.7$ \\
$4-10^{\circ}$ & $558.0 \pm 1.2$ & $43.1 \pm 0.3$ \\
\hline
\end{tabular}

TABLE 1

BACKGround LeVels in $10^{-6} \mathrm{PSPC}_{\text {COUNTS S }}^{-1}$ ARCMIN $^{-2}$.

\begin{tabular}{l|cc}
\hline $\begin{array}{l}\text { Region } \\
\left({ }^{\circ}\right)\end{array}$ & $\begin{array}{c}k T \\
(\mathrm{keV})\end{array}$ & $\begin{array}{c}\mathrm{R} 2 / \mathrm{R} 7 \\
\mathrm{c} / \mathrm{r} \text { ratio }\end{array}$ \\
\hline $0-0.5$ & 8 & 1.04 \\
$0.5-2$ & 4 & 1.28 \\
$2-4$ & 2 & 1.80 \\
\hline
\end{tabular}

TABLE 2

Average temperature of the Coma plasma, and ratio of R2-TO-R7 COUNT RATE FOR PSPC.

We estimate the ratio of $\mathrm{R} 2$-to- $\mathrm{R} 7$ band count rates using the method described in Snowden et al. (1997), which consists of using the on-axis $P S P C$ response function in conjunction with an optically thin plasma emission model ${ }^{5}$. The distribution of Galactic HI in the direction of Coma was investigated in Bonamente et al. (2003), by means of the Dickey \& Lockman (1990) and Hartmann \& Burton (1997) 21-cm data, and IRAS 100 $\mu \mathrm{m}$ data. For a region within $5^{\circ}$ from the cluster center, the HI column density is between $N_{H}=0.8-1.1 \times 10^{20}$ $\mathrm{cm}^{-2}$, with no evidence of large-scale gradients; for the count-rate ratios in Table 2, we assumed $N_{H}=0.9 \times 10^{20}$ $\mathrm{cm}^{-2}$, which is appropriate for the radial range of interest. The count-rate ratio is also sensitive to the chemical composition of the plasma; in particular, a higher abundance $A$ of metals results in a lower R2-to-R7 ratio, due to emission lines processes. We therefore used a conservative value of $A=0$ in deriving the estimates of Table 2, if an abundance of $A=0.3$ was used instead, the count-rate ratio would decrease respectively to 1.03 (8 keV), $1.20(4 \mathrm{keV})$ and $1.48(2 \mathrm{keV})$, causing a lower prediction for the contribution of the hot ICM in the R2 band and higher soft X-ray fluxes. For each annulus, we thus estimate the hot ICM contribution to the $1 / 4 \mathrm{keV}$ band by multiplication of the background-subtracted R7 intensity (Figure 2) by the factor in Table 2.

In Figure 3 we show the R2 band excess emission above the contribution of the hot ICM. This determination of the excess emission clearly depends on our choices in the modelling of the thermal plasma. If the plasma temperature in the $0.5-2^{\circ}$ region is higher, then our estimates are a strict lower limit. The excess emission is still detected at high significance by using a temperature as low as 2 $\mathrm{keV}$ for the $0.5-2^{\circ}$ region; in this case, the significance of detection of the three bins in Figure 3 between 0.5 and $2^{\circ}$ decreases, respectively, from 7.0, 8.0 and $6.1 \sigma$ to $4.2,6.9$ and $5.1 \sigma$. The uncertainty in the R2 excess takes into account the error in the background measurements from the $4-6^{\circ}$ region. As noted above in Section 2.3 , the excess is detected with same statistical significance if the $4-10^{\circ}$ region is used to estimate the background. The increase in the $1 / 4 \mathrm{keV}$ band flux at radii $\geq 8^{\circ}$ is associated with a North Polar Spur feature clearly visible in Figure 1 ,

5 This procedure was also confirmed by S. Snowden, private communication. The PSPC-C camera was used for the All-Sky Survey. and gives rise to a spurious $1 / 4 \mathrm{keV}$ excess. This emission was the reason for our choice of the local background in the $4-6^{\circ}$ region - which is less affected by the North Polar Spur emission- and highlights the need of a local background for the purpose of background subtraction.

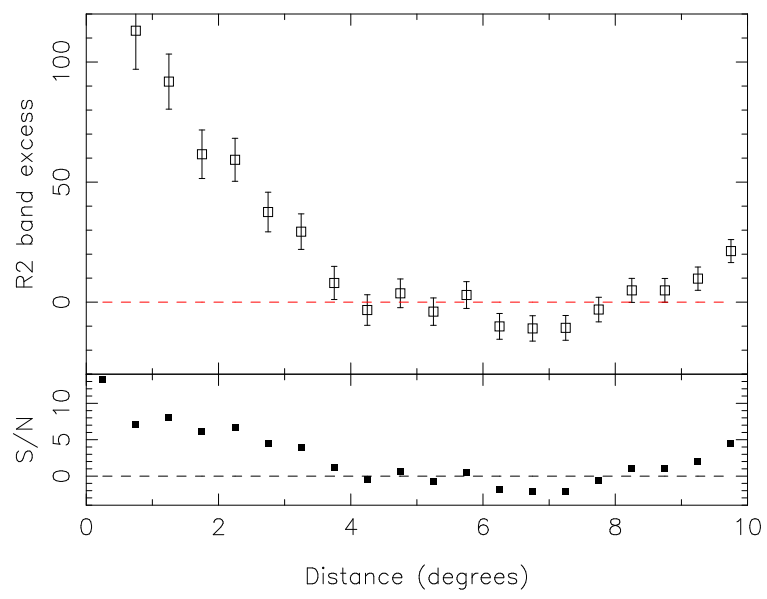

FIG. 3.- Radial profile of the excess emission in R2 band, above the contribution from the hot ICM . Units of the excess emissions are $10^{-6} \mathrm{PSPC}$ counts $\mathrm{s}^{-1} \operatorname{arcmin}^{-2}$.

\section{INTERPRETATION OF THE EXCESS EMISSION}

In Section 2 we discussed possible sources of systematic error that may affect the excess emission shown in Figure 3, and concluded that the signal cannot be explained by errors in the HI column density, modelling of the hot plasma, or background subtraction. We therefore proceed to investigate if the emission can be due to unresolved point sources, and then discuss two scenarios for a cluster origin of the emission.

\subsection{Point source emission}

Given the limited angular resolution of these ROSAT All-Sky Survey data, a possible explanation for the detected excess is that the signal is associated with a number of unresolved point sources (e.g., galaxies) in the Coma field. We address this possibility by using the analysis of X-ray point sources detected by Finoguenov et al. (2004) in an XMM-Newton mosaic observation of the central Coma cluster with the XMM-Newton EPIC detector. Their analysis detects a number of X-ray point sources for a total combined flux of $F=0.67 \pm 0.01 \mathrm{EPIC}$ counts per second in the 1-2 keV band, in a circular area of approximate radius $0.78^{\circ}$. We can use these numbers to estimate the contribution of unresolved point sources in our ROSAT All-Sky Survey data. First, we rescale the EPIC count rate to the PSPC count rate in the same energy band, taking into account that the PSPC camera has an average effective area that is $\sim 7.5 \%$ that of the EPIC detectors, in the $1-2 \mathrm{keV}$ band (Finoguenov et al. 2004; Snowden et al. 1997). Then, assuming (conservatively) that an equivalent population of X-ray sources is present at larger radii, we can estimate that the average brightness due to point sources in the R7 band is of order $S=7.2 \times 10^{-6}$ PSPC counts per second per square arcmin. The contribution of these point sources to the 
R2 band depends on their spectrum; for an X-ray thermal spectrum with $k T=1 \mathrm{keV}$, typical of galaxies, the $\mathrm{R} 2$ count rate is approximately $2-2.5$ times that in the $\mathrm{R} 7$ band, i.e., $\sim 1 \times$ higher than the R2-to-R7 conversion factor used at radii $\geq 0.5^{\circ}$ in converting the $\mathrm{R} 7$ emission to the R2 band (see Table 2). Unresolved point sources would therefore reduce the soft excess fluxes of Figure 3 by approximately $7.2 \times 10^{-6} \mathrm{PSPC}$ counts per second per square arcmin, which corresponds to $10 \%$ of the detected excess at radii less than $2^{\circ}$, and by $10-20 \%$ in the $2-3^{\circ}$ region. We therefore conclude that unresolved point source do not contribute significantly, if at all, to the detected signal.

\subsection{Diffuse excess emission associated with the Coma cluster}

The only remaining possibility is that the detected signal is a truly diffuse celestial component related to the Coma cluster. These data therefore constitute the detection of soft X-ray excess emission out to a radial distance of approximately $3^{\circ}$, or $5 \mathrm{Mpc}$, from the center of the Coma cluster. The main feature of the excess emission is its persistence beyond the radius where the hot ICM is detected $\left(\leq 2^{\circ}\right)$, thus providing strong evidence of the fact that the excess component is unrelated to the thermal plasma. We propose two possible explanations for this large-scale emission.

The first explanation is that the emission is due to Compton scattering of CMB photons off of relativistic electrons, which have been accelerated at locations where gas accreting into the cluster potential becomes supersonic, thus exciting shock waves. The radio observations of Bagchi et al. (2006) show a very spectacular detection of a ring of emission at a distance of $\sim 1 \mathrm{Mpc}$ from the center of the cluster Abell 3376, which is interpreted as the result of accretion shocks. There is theoretical (e.g., Cen \& Ostriker 1999; Davé et al. 2001; Dolag et al. 2006 ) and observational evidence (e.g., Finoguenov et al. 2003; Werner et al. 2008) that diffuse filaments at $k T \simeq$ $10^{5}-10^{7} \mathrm{~K}$ are present in the inter-cluster medium; these structures are therefore the ideal candidate for providing gas accreting into the deeper cluster potential. Shock acceleration, requires the presence of magnetic fields, in order to generate Alfvèn waves that act as scattering centers (e.g. Bell 1978). Magnetic fields are clearly present in Abell 3376, as revealed by the non-thermal radio emission; it is however not clear, though, how magnetic fields would arise in an environment where the density of gas is expected to be very low $\left(\leq 10^{-5} \mathrm{~cm}^{-3}\right)$.

Another interpretation is that optically-thin soft X-ray emission from the filaments themselves is responsible for the detected emission. This scenario has been discussed in several prior papers (e.g., Bonamente et al. 2003; Mittaz et al. 2004; Bonamente et al. 2005), in which it was found that the column density of the filaments would exceed the expectations based on numerical simulations. We use the present ROSAT All-Sky Survey data to estimate the characteristics of the filaments that may be responsible for the excess emission. We assume filaments at a temperature of $k T=0.1 \mathrm{keV}$, and with null abundance of metals, typical of intergalactic filaments, and use the APEC emission code (Smith et al. 2001) to calculate the emissivity as $\Lambda=4.5 \times 10^{-16}$ counts $\mathrm{cm}^{3} \mathrm{~s}^{-1}$ in the $0.14-0.284 \mathrm{keV}$ (R2) band. We also assume a uniform electron density of $n=10^{-4} \mathrm{~cm}^{-3}$, corresponding to a baryonic overdensity of $\delta \sim 200-300$ (for $H_{0}=73$ $\mathrm{km} \mathrm{s}^{-1} \mathrm{Mpc}^{-1}$ ), an average filament length of $L=5$ $\mathrm{Mpc}$ in the direction of the observer. Using the average R2 band effective area of the PSPC detector $\left(A_{\text {eff }} \simeq 150\right.$ $\mathrm{cm}^{2}$ ) the $\mathrm{R} 2$ band surface brightness is calculated as

$S_{X}=\frac{1}{4 \pi} \Lambda \cdot n^{2} L \cdot A_{e f f}=70 \times 10^{-6} \quad\left(\right.$ counts s$\left.{ }^{-1} \operatorname{arcmin}^{-2}\right)$

Comparison with Figure 3 shows that this is in fact the typical excess emission detected at the outskirts of the Coma cluster (radii $\gtrsim 1.5^{\circ}$ ). Since the surface brightness is proportional to $n^{2} L$, lower density filaments would require to be substantially longer along the sightline, in order to explain the detected emission. Filaments of this density or length are more massive than typical filaments in numerical simulations (e.g., Mittaz et al. 2004). A possible explanation for the variance between observations and numerical models is that the filaments are actually magnified by gravitational lensing caused by the cluster potential, as discussed in Lieu and Bonamente (2009, submitted).

An additional interpretation can be provided by the Prokhorov (2008) model in which the soft excess emission is the result of a non-equilibrium state between electrons and ions present at the cluster's outskirts. The model predicts soft X-ray excess from low-temperature electrons near the virial radius, which is approximately $2-3^{\circ}$ (see Section 2.3). These ROSAT All-Sky Survey observations are compatible with such scenario.

\section{DISCUSSION AND CONCLUSIONS}

These ROSAT All-Sky Survey data provide a unique view of the soft excess emission from the Coma cluster, which is now detected out to a $\sim 5 \mathrm{Mpc}$ radius. Diffuse filaments of warm gas provide the natural interpretation for the excess emission, either via particle acceleration at accretion shocks, or simply via their thermal emission. The scenario of thermal emission from filaments was tested using XMM-Newton observations of Abell S1101 (Bonamente et al. 2005); in that case, we determined that the filaments would be required to be even more massive than the ones discussed in this paper (for the same nominal density $n=10^{-4} \mathrm{~cm}^{-3}$, the filament length would exceed $10 \mathrm{Mpc}$ ). In an upcoming paper (in preparation), we will show that the excess emission in Abell S1101 was overestimated, due to a significant revision of the HI column density in the direction to the cluster (Kalberla et al. 2005); the reduced flux will make the estimates similar to the ones presented in this paper.

Current X-ray missions are not designed for studies of large-scale soft X-ray emission, given their narrow field of view and issues in the calibration of the $1 / 4$ $\mathrm{keV}$ band (see Nevalainen et al. 2007 for a discussion on the XMM-Newton soft band, and Bonamente et al. 2007 on Chandra). The presence of filaments has therefore been mainly investigated in absorption, giving few tentative detections of absorption lines due to filaments. In particular, Nicastro et al. (2005) detected $z>0$ absorption lines towards Markarian 421 with Chandra, although Rasmussen et al. (2007) did not confirm the de- 
tection with XMM-Newton. Of particular relevance to the Coma excess is the work of Takei et al. (2007), who used XMM-Newton RGS data to obtain a 3- $\sigma$ detection of absorption lines in the spectrum of X Comae, a background quasar at a projected distance $\sim 25^{\prime}$ from the cluster center. Although that detection is of limited statistical significance, it provides support to the thermal interpretation of the soft excess emission we discussed in Section 2.4, at least in the central regions of the cluster.

The ROSAT mission continues to provide the most compelling detections of soft excess emission from galaxy clusters. Using pointed $P S P C$ data, we were initially able to detect soft excess emission in several clusters (Bonamente et al. 2001a,, b) , confirming the discovery papers based on EUVE observation (Lieu et al. 1996a b). We then showed that a large fraction of clusters at high Galactic latitude feature the excess emission (Bonamente et al. 2002), used a mosaic of four pointed observations of Coma to detect the emission out to 2.6 Mpc (Bonamente et al. 2003) and now, based on the ROSAT All-Sky Survey data, out to 5 Mpc. In all cases, ROSAT's unique combination of a reliable calibration in the $1 / 4 \mathrm{keV}$ band, and the availability of contemporaneous background, rendered these detections possible. The main advantage of searching for filaments in emission is the insensitivity to the abundance of metals, and to clumping of the gas. The observations analyzed in this paper show that a very modest exposure time with a wide-field soft X-ray camera is a most efficient way to detect emission from warm filaments in the neighborhood of clusters.

We are grateful to Dr. S. Snowden for comments and suggestions on the data analysis.

\section{REFERENCES}

Arnaud, M., Aghanim, N., Gastaud, R., Neumann, D. M., Lumb, D., Briel, U., Altieri, B., Ghizzardi, S., Mittaz, J., Sasseen, T. P., \& Vestrand, W. T. 2001, A\&A, 365, L67

Bagchi, J., Durret, F., Neto, G. B. L., \& Paul, S. 2006, Science, 314, 791

Bell, A. R. 1978, MNRAS, 182, 147

Beuermann, K., Burwitz, V., \& Rauch, T. 2006, A\&A, 458, 541

Bonamente, M., Joy, M. K., \& Lieu, R. 2003, ApJ, 585, 722

Bonamente, M., Lieu, R., Joy, M. K., \& Nevalainen, J. H. 2002, ApJ, 576, 688

Bonamente, M., Lieu, R., \& Mittaz, J. P. D. 2001a, ApJ, 561, L63 -. 2001b, ApJ, 547, L7

Bonamente, M., Lieu, R., Mittaz, J. P. D., Kaastra, J. S., \& Nevalainen, J. 2005, ApJ, 629, 192

Bonamente, M., Lieu, R., Nevalainen, J., \& Kaastra, J. S. 2001c, ApJ, 552, L7

Bonamente, M., Nevalainen, J., \& Lieu, R. 2007, ApJ, 668, 796

Bowyer, S., Berghöfer, T. W., \& Korpela, E. J. 1999, ApJ, 526, 592

Bowyer, S., Korpela, E. J., Lampton, M., \& Jones, T. W. 2004, ApJ, 605, 168

Briel, U. G., Henry, J. P., \& Boehringer, H. 1992, A\&A, 259, L31

Brunetti, G., Venturi, T., Dallacasa, D., Cassano, R., Dolag, K., Giacintucci, S., \& Setti, G. 2007, ApJ, 670, L5

Cavagnolo, K. W., Donahue, M., Voit, G. M., \& Sun, M. 2008, ApJ, 682, 821

Cen, R. \& Ostriker, J. P. 1999, ApJ, 514, 1

Cheng, L.-M., Borgani, S., Tozzi, P., Tornatore, L., Diaferio, A., Dolag, K., He, X.-T., Moscardini, L., Murante, G., \& Tormen, G. $2005, A \& A, 431,405$

Clarke, T. E. \& Ensslin, T. A. 2006, AJ, 131, 2900

Davé, R., Cen, R., Ostriker, J. P., Bryan, G. L., Hernquist, L., Katz, N., Weinberg, D. H., Norman, M. L., \& O'Shea, B. 2001, ApJ, 552, 473

Dickey, J. M. \& Lockman, F. J. 1990, ARA\&A, 28, 215

Dolag, K., Meneghetti, M., Moscardini, L., Rasia, E., \& Bonaldi, A. 2006, MNRAS, 370, 656

Durret, F., Kaastra, J. S., Nevalainen, J., Ohashi, T., \& Werner, N. 2008, Space Science Reviews, 134, 51

Feretti, L., Fusco-Femiano, R., Giovannini, G., \& Govoni, F. 2001, A\&A, 373, 106

Finoguenov, A., Briel, U. G., \& Henry, J. P. 2003, A\&A, 410, 777

Finoguenov, A., Briel, U. G., Henry, J. P., Gavazzi, G., Iglesias-Paramo, J., \& Boselli, A. 2004, A\&A, 419, 47

Finoguenov, A., Reiprich, T. H., \& Böhringer, H. 2001, A\&A, 368,749

Garmire, G. P., Nousek, J. A., Apparao, K. M. V., Burrows, D. N., Fink, R. L., \& Kraft, R. P. 1992, ApJ, 399, 694

Geller, M. J., Diaferio, A., \& Kurtz, M. J. 1999, ApJ, 517, L23
Hartmann, D. \& Burton, W. B. 1997, Atlas of Galactic Neutral Hydrogen (Atlas of Galactic Neutral Hydrogen, by Dap Hartmann and W. Butler Burton, pp. 243. ISBN 0521471117. Cambridge, UK: Cambridge University Press, February 1997.)

Hughes, J. P. 1989, ApJ, 337, 21

Kalberla, P. M. W., Burton, W. B., Hartmann, D., Arnal, E. M., Bajaja, E., Morras, R., \& Pöppel, W. G. L. 2005, A\&A, 440, 775

Kubo, J. M., Stebbins, A., Annis, J., Dell'Antonio, I. P., Lin, H., Khiabanian, H., \& Frieman, J. A. 2007, ApJ, 671, 1466

Lieu, R., Ip, W.-H., Axford, W. I., \& Bonamente, M. 1999, ApJ, 510, L 25

Lieu, R., Mittaz, J. P. D., Bowyer, S., Breen, J. O., Lockman, F. J., Murphy, E. M., \& Hwang, C.-Y. 1996a, Science, 274, 1335

Lieu, R., Mittaz, J. P. D., Bowyer, S., Lockman, F. J., Hwang, C.-Y., \& Schmitt, J. H. M. M. 1996b, ApJ, 458, L5+

Łokas, E. L. \& Mamon, G. A. 2003, MNRAS, 343, 401

Lutovinov, A. A., Vikhlinin, A., Churazov, E. M., Revnivtsev, M. G., \& Sunyaev, R. A. 2008, ApJ, 687, 968

McCammon, D., Burrows, D. N., Sanders, W. T., \& Kraushaar, W. L. 1983, ApJ, 269, 107

Mittaz, J., Lieu, R., Cen, R., \& Bonamente, M. 2004, ApJ, 617, 860

Nevalainen, J., Bonamente, M., \& Kaastra, J. 2007, ApJ, 656, 733

Nevalainen, J., Lieu, R., Bonamente, M., \& Lumb, D. 2003, ApJ, 584,716

Nicastro, F., Mathur, S., Elvis, M., Drake, J., Fiore, F., Fang, T., Fruscione, A., Krongold, Y., Marshall, H., \& Williams, R. 2005, ApJ, 629, 700

Pfeffermann, E., Briel, U. G., Hippmann, H., Kettenring, G., Metzner, G., Predehl, P., Reger, G., Stephan, K.-H., Zombeck, M., Chappell, J., \& Murray, S. S. 1987, in Society of Photo-Optical Instrumentation Engineers (SPIE) Conference Series, Vol. 733, Society of Photo-Optical Instrumentation Engineers (SPIE) Conference Series, ed. E.-E. Koch \& G. Schmahl, 519-+

Prokhorov, D. A. 2008, A\&A, 492, 651

Rasmussen, A. P., Kahn, S. M., Paerels, F., Herder, J. W. d., Kaastra, J., \& de Vries, C. 2007, ApJ, 656, 129

Sarazin, C. L. \& Lieu, R. 1998, ApJ, 494, L177+

Smith, R. K., Brickhouse, N. S., Liedahl, D. A., \& Raymond, J. C. 2001, ApJ, 556, L91

Snowden, S. L., Egger, R., Freyberg, M. J., McCammon, D., Plucinsky, P. P., Sanders, W. T., Schmitt, J. H. M. M., Truemper, J., \& Voges, W. 1997, ApJ, 485, 125

Snowden, S. L., Freyberg, M. J., Plucinsky, P. P., Schmitt, J. H. M. M., Truemper, J., Voges, W., Edgar, R. J., McCammon, D., \& Sanders, W. T. 1995, ApJ, 454, 643

Takei, Y., Henry, J. P., Finoguenov, A., Mitsuda, K., Tamura, T., Fujimoto, R., \& Briel, U. G. 2007, ApJ, 655, 831 
Takei, Y., Miller, E. D., Bregman, J. N., Kimura, S., Ohashi, T., Mitsuda, K., Tamura, T., Yamasaki, N. Y., \& Fujimoto, R.

2008, ApJ, 680, 1049

Vikhlinin, A., Kravtsov, A., Forman, W., Jones, C., Markevitch, M., Murray, S. S., \& Van Speybroeck, L. 2006, ApJ, 640, 691

Werner, N., Finoguenov, A., Kaastra, J. S., Simionescu, A.,

Dietrich, J. P., Vink, J., \& Böhringer, H. 2008, A\&A, 482, L29
Werner, N., Kaastra, J. S., Takei, Y., Lieu, R., Vink, J., \& Tamura, T. 2007, A\&A, 468, 849 